\title{
Motor Development
}

National Cancer Institute

\section{Source}

National Cancer Institute. Motor Development. NCI Thesaurus. Code C81269.

The evolution of physical movement over an individual's lifespan. 